Copyright (C) 2014 IEEE. Personal use of this material is permitted. Permission from IEEE must be obtained for all other uses, in any current or future media, including reprinting/republishing this material for advertising or promotional purposes, creating new collective works, for resale or redistribution to servers or lists, or reuse of any copyrighted component of this work in other works. 


\title{
A gradient-based parameter identification method for time-delay chaotic systems
}

\author{
Qinqin CHAI ${ }^{1}$, Ryan LOXTON ${ }^{2}$ \\ 1. College of Electrical Engineering and Automation, Fuzhou University, Fuzhou, 350108, People's Republic of China \\ E-mail: kppqing@163.com \\ 2. Department of Mathematics and Statistics, Curtin University, Perth, 6102, Australia \\ E-mail: r.loxton@curtin.edu.au
}

\begin{abstract}
In this paper, the parameter identification problem for a general class of time-delay chaotic systems is considered. The objective of the problem is to determine optimal values for an unknown time-delay and unknown system parameters such that the dynamic model of the system best fits given experimental data. We propose a gradient-based optimization algorithm to solve this problem, where accurate values for the partial derivatives of the error function are obtained by solving a set of auxiliary time-delay systems. Simulation results for two example problems show that the proposed algorithm is robust and efficient.
\end{abstract}

Key Words: Chaotic system, Parameter identification, Time-delay

\section{Introduction}

Time-delays are present in many real-word chaotic systems, including chemical processes [1], biological system$\mathrm{s}$ [2], economic systems [3], and secure communication systems [4]. Sometimes the time-delays appear naturally; sometimes they are deliberately introduced to help stabilize the system or force the system to track real data. The control and synchronization of chaotic systems is now an active area of research. A key result shows that, under an appropriate control law, the output of a chaotic system can be made to follow any given reference signal [7]. This properly is widely used in the control and optimization of chaotic systems.

Chaotic systems with time-delays have attracted particular attention in the literature. For example, some popular approaches for solving the synchronization problem for time-delay chaotic systems include the delay-dependent synchronization method based on the Lyapunov-Krasovskii function [5], the adaptive fuzzy sliding mode control approach [6], and the active control approach [8]. Most of the existing control and synchronization methods for time-delay chaotic systems are only effective when all model parameters are known. However, in practice, it is common for the values of some model parameters (and even the magnitudes of the time-delays) to be unknown. Thus, before designing a control scheme to realize synchronization, the unknown parameters (including the time-delay) must first be identified.

Parameter identification problems for chaotic systems have attracted increasing attention in recent years. However, there are many open issues yet to be resolved. For example, although swarm intelligence optimization methods (such as seeker optimization algorithms, particle swarm optimization algorithms, differential evolution algorithms) are popular [9-11], they are often unstable when applied to chaotic systems. This is due to the complexity and volatile nature of chaotic systems. On the other hand, although gradientbased optimization techniques have excellent local searching capabilities, they have yet to be fully exploited to solve

This work was supported by the National Natural Science Foundation of China through the International Young Scientists Research Fellowship program (Grant 11350110208), and the Research Foundation of Fuzhou University (Grant 510050). the parameter identification problem for time-delay chaotic systems. In addition, the existing identification methods for nonlinear time-delay systems (see [12-17]) are not suitable for chaotic systems, as such systems typically contain nonlinear terms with more than one unknown delay/parameter.

In this paper, we consider the problem of identifying an unknown time-delay and unknown model parameters in a general time-delay chaotic system. We will propose a gradient-based computational method to solve this parameter identification problem and thus determine the optimal delay/parameter values that minimize estimation error. The remainder of the paper is organized as follows. The parameter identification problem is formulated in Section 2. In Section 3, our computational method, which is based on exploiting the gradient of the error function, is developed. Two examples are then solved in Section 4. Section 5 concludes the paper.

\section{Problem Formulation}

Consider a time-delay chaotic system described by the following delay-differential equation:

$$
\begin{aligned}
& \dot{\boldsymbol{x}}(t)=\boldsymbol{f}(t, \boldsymbol{x}(t), \boldsymbol{x}(t-\tau), \boldsymbol{\zeta}), \quad t \in[0, T], \\
& \boldsymbol{x}(t)=\boldsymbol{\phi}(t), \quad t \leq 0,
\end{aligned}
$$

where $T>0$ is a given terminal time; $\boldsymbol{x}(t)=$ $\left[x_{1}(t), \ldots, x_{n}(t)\right]^{\top} \in \mathbb{R}^{n}$ is the state vector; $\tau$ is an unknown time-delay; and $\zeta=\left[\zeta_{1}, \ldots, \zeta_{r}\right]^{\top} \in \mathbb{R}^{r}$ is a vector of unknown system parameters. Furthermore, $f: \mathbb{R} \times \mathbb{R}^{n} \times$ $\mathbb{R}^{n} \times \mathbb{R}^{r} \rightarrow \mathbb{R}^{n}$ and $\phi: \mathbb{R} \rightarrow \mathbb{R}^{n}$ are given functions. We assume that $f$ is continuously differentiable and $\phi$ is twice continuously differentiable.

The time-delay and system parameters in (1)-(2) are unknown quantities that need to be determined. We assume that they satisfy the following bound constraints:

$$
\begin{aligned}
a & \leq \tau \leq b, \\
c_{j} & \leq \zeta_{j} \leq d_{j}, \quad j=1, \ldots, r,
\end{aligned}
$$

where $a$ and $b$ are given constants such that $0 \leq a<b$, and $c_{j}$ and $d_{j}$ are given constants such that $0 \leq c_{j}<d_{j}$. 
Any scalar $\tau$ that satisfies (3) is referred to as an admissible time-delay. Let $\mathcal{T}$ denote the set of all such admissible time-delays. Any vector $\zeta=\left[\zeta_{1}, \ldots, \zeta_{r}\right]^{\top} \in \mathbb{R}^{r}$ that satisfies (4) is referred to as an admissible parameter vector. Let $\mathcal{Z}$ denote the set of all such admissible parameter vectors. Any pair $(\tau, \boldsymbol{\zeta}) \in \mathcal{T} \times \mathcal{Z}$ is called a candidate solution pair.

We assume that the following condition is satisfied.

Assumption 1. There exists a real number $L_{1}>0$ such that

$$
\begin{aligned}
|\boldsymbol{f}(t, \boldsymbol{x}, \tilde{\boldsymbol{x}}, \boldsymbol{\zeta})| & \leq L_{1}(1+|\boldsymbol{x}|+|\tilde{\boldsymbol{x}}|+|\boldsymbol{\zeta}|), \\
& (t, \boldsymbol{x}, \tilde{\boldsymbol{x}}, \boldsymbol{\zeta}) \in[0, T] \times \mathbb{R}^{n} \times \mathbb{R}^{n} \times \mathbb{R}^{r},
\end{aligned}
$$

where $|\cdot|$ denotes the Euclidean norm.

Assumption 1 ensures that the dynamic system (1)-(2) admits a unique solution corresponding to each candidate solution pair $(\tau, \zeta) \in \mathcal{T} \times \mathcal{Z}$ [18]. We denote this solution by $\boldsymbol{x}(\cdot \mid \tau, \boldsymbol{\zeta})$.

Suppose that the state from system (1)-(2) has been measured experimentally at times $t=t_{l}, l=1, \ldots, q$, where each $t_{l} \in[0, T]$. Let $\hat{\boldsymbol{x}}\left(t_{l}\right) \in \mathbb{R}^{n}$ denote the measured state at time $t=t_{l}$. To identify the unknown time-delay and unknown system parameters, we need to minimize the discrepancy between the measured state values $\hat{\boldsymbol{x}}\left(t_{l}\right), l=1, \ldots, q$, and the estimated state trajectory generated from the dynamic model (1)-(2). This problem can be formulated mathematically as follows.

Problem P. Given system (1)-(2), choose a candidate solution pair $(\tau, \zeta) \in \mathcal{T} \times \mathcal{Z}$ to minimize the following error function:

$$
J(\tau, \boldsymbol{\zeta})=\sum_{l=1}^{q}\left|\boldsymbol{x}\left(t_{l} \mid \tau, \boldsymbol{\zeta}\right)-\hat{\boldsymbol{x}}\left(t_{l}\right)\right|^{2}
$$

\section{Solution Procedure}

Chaotic systems in the form of (1)-(2) are often highly nonlinear and exhibit unstable behavior. Thus, it is difficult to estimate values for the time-delay and model parameters using swarm intelligence optimization methods. In principle, Problem $\mathrm{P}$ is a nonlinear dynamic optimization problem with multiple characteristic times at $t=t_{l}, l=1, \ldots, q$ [19]. Such problems can be viewed as nonlinear programming problems that involve choosing a finite number of decision variables to minimize a given objective function. Thus, standard gradient-based optimization algorithms [20] can be used to solve Problem P. However, to do so, obtaining accurate gradient information is essential. This is not an easy task because the unknown delay and model parameters influence the error function (5) implicitly through the dynamic system (1)-(2). We previously considered this type of parameter identification problem for non-chaotic systems in [21]. In this section, we will develop a computational algorithm for solving Problem $\mathrm{P}$ based on the gradient computation procedure pioneered in [21].

\subsection{Gradient Computation}

The partial derivatives of $J$ with respect to the time-delay and system parameters can be obtained according to the following results given in [21].
Theorem 1. Let $t \in(0, T]$ be a fixed time point. Then $\boldsymbol{x}(t \mid \cdot, \cdot)$ is differentiable with respect to $\tau$ on $\mathcal{T} \times \mathcal{Z}$. In fact, for each $(\tau, \zeta) \in \mathcal{T} \times \mathcal{Z}$,

$$
\frac{\partial \boldsymbol{x}(t \mid \tau, \boldsymbol{\zeta})}{\partial \tau}=\boldsymbol{\Lambda}(t \mid \tau, \boldsymbol{\zeta})
$$

with $\Lambda(\cdot \mid \tau, \zeta)$ defined as the solution of the auxiliary timedelay system

$$
\begin{aligned}
\dot{\boldsymbol{\Lambda}}(t)= & \frac{\partial \boldsymbol{f}(t, \boldsymbol{x}(t), \boldsymbol{x}(t-\tau), \boldsymbol{\zeta})}{\partial \boldsymbol{x}} \boldsymbol{\Lambda}(t) \\
& +\frac{\partial \boldsymbol{f}(t, \boldsymbol{x}(t), \boldsymbol{x}(t-\tau), \boldsymbol{\zeta})}{\partial \boldsymbol{x}(t-\tau)} \boldsymbol{\Lambda}(t-\tau) \\
& -\frac{\partial \boldsymbol{f}(t, \boldsymbol{x}(t), \boldsymbol{x}(t-\tau), \boldsymbol{\zeta})}{\partial \boldsymbol{x}(t-\tau)} \boldsymbol{\chi}(t-\tau)
\end{aligned}
$$

with initial condition

$$
\boldsymbol{\Lambda}(t)=\mathbf{0}, \quad t \leq 0,
$$

where

$$
\chi(t)= \begin{cases}\dot{\phi}(t), & \text { if } t \leq 0, \\ \boldsymbol{f}(t, \boldsymbol{x}(t), \boldsymbol{x}(t-\tau), \boldsymbol{\zeta}), & \text { if } t \in(0, T]\end{cases}
$$

Theorem 2. Let $t \in(0, T]$ be a fixed time point. Then $\boldsymbol{x}(t \mid \cdot, \cdot)$ is differentiable with respect to $\zeta_{j}$ on $\mathcal{T} \times \mathcal{Z}$. In fact, for each $(\tau, \zeta) \in \mathcal{T} \times \mathcal{Z}$,

$$
\frac{\partial \boldsymbol{x}(t \mid \tau, \boldsymbol{\zeta})}{\partial \zeta_{j}}=\boldsymbol{\Gamma}^{j}(t \mid \boldsymbol{\tau}, \boldsymbol{\zeta}), \quad j=1, \ldots, r
$$

with $\Gamma^{j}(\cdot \mid \tau, \zeta)$ defined as the solution of the auxiliary timedelay system

$$
\begin{aligned}
\dot{\boldsymbol{\Gamma}}^{j}(t)= & \frac{\partial \boldsymbol{f}(t, \boldsymbol{x}(t), \boldsymbol{x}(t-\tau), \boldsymbol{\zeta})}{\partial \boldsymbol{x}} \boldsymbol{\Gamma}^{j}(t) \\
& +\frac{\partial \boldsymbol{f}(t, \boldsymbol{x}(t), \boldsymbol{x}(t-\tau), \boldsymbol{\zeta})}{\partial \boldsymbol{x}(t-\tau)} \boldsymbol{\Gamma}^{j}(t-\tau) \\
& +\frac{\partial \boldsymbol{f}(t, \boldsymbol{x}(t), \boldsymbol{x}(t-\tau), \boldsymbol{\zeta})}{\partial \zeta_{j}}
\end{aligned}
$$

with initial condition

$$
\boldsymbol{\Gamma}^{j}(t)=\mathbf{0}, \quad t \leq 0 .
$$

See [21] for more details regarding Theorems 1 and 2.

By using Theorems 1 and 2 and the chain rule of differentiation, the gradient formulae for the error function (5) in Problem P can be derived as follows:

$$
\frac{\partial J(\tau, \boldsymbol{\zeta})}{\partial \tau}=2 \sum_{l=1}^{q}\left[\boldsymbol{x}\left(t_{l} \mid \tau, \boldsymbol{\zeta}\right)-\hat{\boldsymbol{x}}\left(t_{l}\right)\right]^{\top} \boldsymbol{\Lambda}\left(t_{l} \mid \tau, \boldsymbol{\zeta}\right),
$$

and

$$
\frac{\partial J(\tau, \boldsymbol{\zeta})}{\partial \zeta_{j}}=2 \sum_{l=1}^{q}\left[\boldsymbol{x}\left(t_{l} \mid \tau, \boldsymbol{\zeta}\right)-\hat{\boldsymbol{x}}\left(t_{l}\right)\right]^{\top} \boldsymbol{\Gamma}^{j}\left(t_{l} \mid \tau, \boldsymbol{\zeta}\right) .
$$




\subsection{Computational Algorithm}

It is well known that chaotic systems are unstable and highly sensitive to the initial state and the values of the model parameters. In addition, gradient-based optimization methods, such as sequential quadratic programming (SQP), are only guaranteed to converge to a locally-optimal solution. Thus, when applied to Problem P, these methods could potentially generate parameter estimates that are not globally optimal (the quality of the delay/parameter values obtained depends on the initial guess). To overcome this drawback, we propose the following approach for generating the initial guess for the gradient-based optimization method.

We first randomly generate $N$ initial candidate solution pairs $\left(\bar{\tau}_{i}, \overline{\boldsymbol{\zeta}}_{i}\right), i=1, \ldots, N$. Then, we compute the corresponding cost values $J_{i}=J\left(\bar{\tau}_{i}, \bar{\zeta}_{i}\right)$. The initial guess for the gradient-based optimization method is chosen as $\left(\bar{\tau}_{k}, \bar{\zeta}_{k}\right)$, where $k=\arg \min \left\{J_{i}: i=1, \ldots, N\right\}$. By performing this initialization procedure, the gradient-based optimization method will start from a good initial approximation of the optimal parameter values, and will therefore have high likelihood of converging to the global optimum. Furthermore, in a practical identification problem, tight bounds are often available for the unknown parameters. Thus, the initial approximations are usually close to the real values. In this case, global optimality can be achieved using gradient-based optimization methods.

We now present the following gradient-based optimization method to solve Problem P.

1. Generate an initial guess $(\tau, \boldsymbol{\zeta})$ according to the procedure described above.

2. Obtain $\boldsymbol{x}(\cdot \mid \tau, \boldsymbol{\zeta}), \boldsymbol{\Lambda}(\cdot \mid \tau, \boldsymbol{\zeta})$ and $\boldsymbol{\Gamma}^{j}(\cdot \mid \tau, \boldsymbol{\zeta}), j=1, \ldots, r$, by solving the enlarged time-delay system consisting of the original system (1)-(2) and the auxiliary systems (6)-(7) and (8)-(9).

3. Use $\boldsymbol{x}\left(t_{l} \mid \tau, \boldsymbol{\zeta}\right), l=1, \ldots, q$, to compute $J(\tau, \boldsymbol{\zeta})$ through equation (5).

4. Use $\boldsymbol{x}\left(t_{l} \mid \tau, \boldsymbol{\zeta}\right), \boldsymbol{\Lambda}\left(t_{l} \mid \tau, \boldsymbol{\zeta}\right)$, and $\boldsymbol{\Gamma}^{j}\left(t_{l} \mid \tau, \boldsymbol{\zeta}\right)$ to compute $\partial J(\tau, \boldsymbol{\zeta}) / \partial \tau$ and $\partial J(\tau, \boldsymbol{\zeta}) / \partial \zeta_{j}, j=1, \ldots, r$, through equations (10) and (11).

5. Use $J(\tau, \boldsymbol{\zeta}), \partial J(\tau, \boldsymbol{\zeta}) / \partial \tau$, and $\partial J(\tau, \boldsymbol{\zeta}) / \partial \zeta_{j}, j=$ $1, \ldots, r$, to find a new solution candidate $\left(\tau^{\prime}, \boldsymbol{\zeta}^{\prime}\right)$ according to a gradient-based optimization procedure (e.g., SQP).

6. If termination conditions are satisfied, then stop. Otherwise, set $\left(\tau^{\prime}, \boldsymbol{\zeta}^{\prime}\right) \rightarrow(\tau, \boldsymbol{\zeta})$ and return to step 2 .

\section{Numerical Examples}

\subsection{Example 1: Logistic Chaotic System}

We consider the parameter identification problem for a time-delay logistic system [11]. This system is described as follows:

$$
\dot{x}(t)=-\zeta_{1} x(t)+\zeta_{2} x(t-\tau)(1-x(t-\tau)), \quad t \in[0, T],
$$

with initial condition

$$
x(t)=\phi(t), \quad t \leq 0,
$$

where $\zeta_{1}$ and $\zeta_{2}$ are unknown system parameters and $\tau$ is an unknown time-delay. These unknown quantities satisfy the following constraints:

$$
22 \leq \zeta_{1} \leq 30, \quad 100 \leq \zeta_{2} \leq 108, \quad 0.2 \leq \tau \leq 1 .
$$

It is known that system (12)-(13) is chaotic when

$$
\zeta_{1}=26, \quad \zeta_{2}=104, \quad \tau=0.5 .
$$

To generate the initial function $\phi$, we simulate the system (12)-(13) with parameter values (15) over the time interval $[0,4]$, where the past time state is assumed to be $x(t)=$ $1, t \leq 0$. Then, we take the resulting state trajectory on $[2,4]$, which is shown as the solid red line in Fig. 1, as the initial function $\phi$. From Fig. 1, we can clearly see the chaotic nature of system (12)-(13).

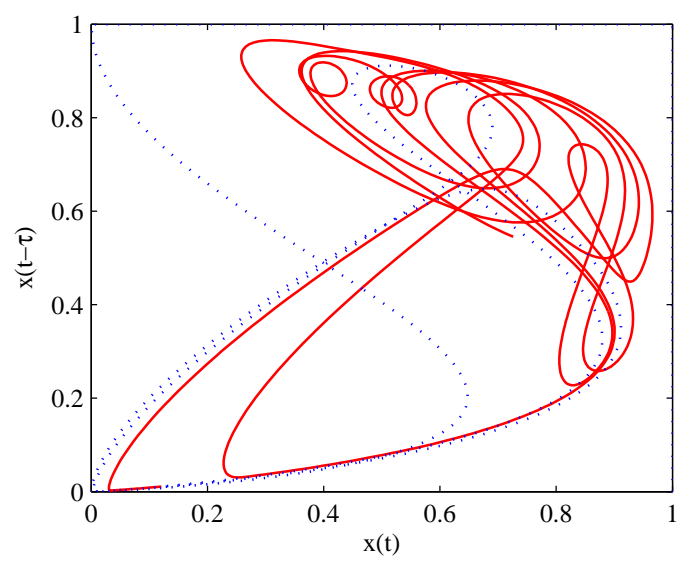

Fig. 1: Solution of system (12)-(13) with parameter values (15) and past state $x(t)=1, t \leq 0$ (the dashed line is the state trajectory on $[0,2]$; the solid line is the state trajectory on $[2,4]$ ).

Starting with the initial state $\phi$, we next generated the observed data in Problem P. This was done by assuming that $T=2$ and simulating (12)-(13) with

$$
\zeta_{1}=\hat{\zeta}_{1}=26, \quad \zeta_{2}=\hat{\zeta}_{2}=104, \quad \tau=\hat{\tau}=0.5 .
$$

The sample times are $t_{l}=l / 5, l=1, \ldots, 10$, and the observed state is $\hat{x}\left(t_{l}\right)=x\left(t_{l} \mid \hat{\zeta}_{1}, \hat{\zeta}_{2}, \hat{\tau}\right)$.

Our parameter identification problem can be stated as follows: Given system (12) with initial conditions (13), choose the unknown quantities $\zeta_{1}, \zeta_{2}$ and $\tau$ to minimize the objective function

$$
J\left(\zeta_{1}, \zeta_{2}, \tau\right)=\sum_{l=1}^{10}\left|x\left(t_{l} \mid \zeta_{1}, \zeta_{2}, \tau\right)-x\left(t_{l} \mid \hat{\zeta}_{1}, \hat{\zeta}_{2}, \hat{\tau}\right)\right|^{2}
$$

subject to constraints (14).

We solved this problem using a Matlab program that integrates the SQP optimization method with the algorithm described in Section 3.2, where the initial guesses for $\zeta_{1}$, $\zeta_{2}$, and $\tau$ are obtained using the randomization method with $N=10$.

Our computational results for this example (including estimated parameters $\left(\zeta_{1}, \zeta 2, \tau\right)$, error function values (EFV) and error function count (EFC)) are reported in Table 1. The convergence of the output trajectory is displayed in Fig. 2. 
We see from Table 1 that the initial guess obtained from the randomization procedure is close to the optimum. Starting from this initial guess, the state trajectory converges quickly to the observed data. Thus, our algorithm easily recovers the true values of the delay and parameters for this problem. In reference [11], this problem was solved using the evolutionary optimization with population size 30 . The evolutionary algorithm requires solving the dynamic system (12)-(13) more than 3000 times. In contrast, using our method, only $218 \times 4=872$ dynamic systems need to be solved. Thus, the computational burden is greatly reduced.

Table 1: Numerical convergence in Example 1.

\begin{tabular}{c|c|c|c|c}
\hline \hline Iteration & 0 & 5 & 15 & 25 \\
\hline$\zeta_{1}$ & 26.723 & 26.524 & 26.262 & 26.000 \\
\hline$\zeta_{2}$ & 104.911 & 104.963 & 105.020 & 104.000 \\
\hline$\tau$ & 0.527 & 0.501 & 0.500 & 0.500 \\
\hline $\mathrm{EFV}$ & 0.224 & $8.8 \times 10^{-3}$ & $3.3 \times 10^{-5}$ & $5.3 \times 10^{-12}$ \\
\hline $\mathrm{EFC}$ & 1 & 17 & 109 & 218 \\
\hline \hline
\end{tabular}

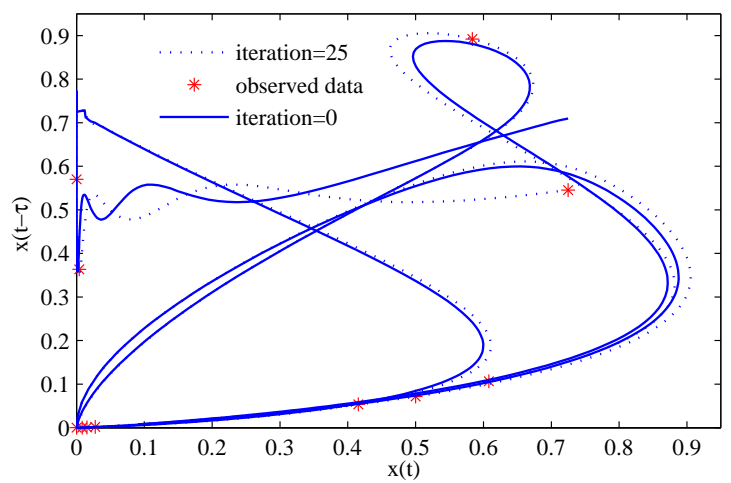

Fig. 2: Convergence of the output trajectory in Example 1.

\subsection{Example 2: Mackey-Glass Chaotic System}

The Mackey-Glass chaotic system is used to model oscillations and chaos occurring in the physiological system related to dynamical respiratory and hematopoietic diseases. This system is defined by the scalar differential equation given as follows [2]:

$$
\dot{x}(t)=-\zeta_{1} x(t)+\frac{\zeta_{2} x(t-\tau)}{1+x(t-\tau)^{m}},
$$

with initial conditions

$$
x(t)=\phi(t), \quad t \leq 0
$$

Here, $m=10$ is a given system parameter; $\zeta_{1}$ and $\zeta_{2}$ are unknown system parameters and $\tau$ is an unknown timedelay. The unknown quantities satisfy the following constraints [11]:

$$
0.05 \leq \zeta_{1} \leq 1, \quad 0.05 \leq \zeta_{2} \leq 1, \quad 12 \leq \tau \leq 20 .
$$

System (17)-(18) is chaotic when

$$
\zeta_{1}=0.1, \quad \zeta_{2}=0.2, \quad \tau=17 .
$$

To generate the initial function $\phi$, we simulated the system (17)-(18) with parameter values (20) over the time interval $[0,400]$, where the past time state is $x(t)=0.5, t \leq 0$. Then, we took the resulting state trajectory on $t \in[350,400]$, which is shown as the solid red line in Fig. 3, as the past time state $\phi$.

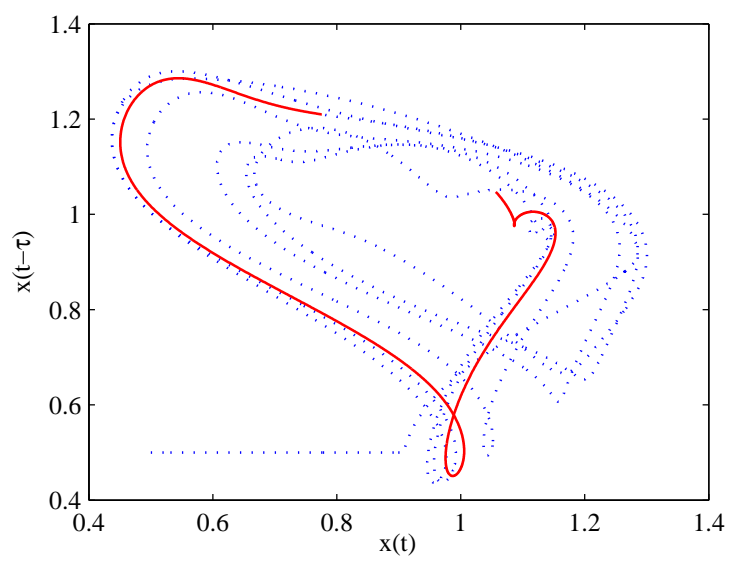

Fig. 3: Solution of system (17)-(18) with parameter values (20) and past state $x(t)=0.5, t \leq 0$ (the dashed line is the state trajectory on $[0,350]$; the solid line is the state trajectory on $[350,400])$.

Starting from the obtained initial state $\phi$, we next generated the observed data in Problem P. This was done by assuming that $T=50$ and simulating (17)-(18) with

$$
\zeta_{1}=\hat{\zeta}_{1}=0.1, \quad \zeta_{2}=\hat{\zeta}_{2}=0.2, \quad \tau=\hat{\tau}=17 .
$$

The sample times are $t_{l}=l / 2, l=1, \ldots, 100$, and the observed state is $\hat{x}\left(t_{l}\right)=x\left(t_{l} \mid \hat{\zeta}_{1}, \hat{\zeta}_{2}, \hat{\tau}\right)$.

Hence, our error function is

$$
J\left(\zeta_{1}, \zeta_{2}, \tau\right)=\sum_{l=1}^{100}\left|x\left(t_{l} \mid \zeta_{1}, \zeta_{2}, \tau\right)-x\left(t_{l} \mid \hat{\zeta}_{1}, \hat{\zeta}_{2}, \hat{\tau}\right)\right|^{2}
$$

The estimation problem is to minimize (21) subject to the bound constraints (19). We solved this problem using the same Matlab program that was used to solve Example 1. The optimization results are shown in Table 2. The convergence of the output trajectory is displayed in Fig. 4.

Table 2: Numerical convergence in Example 2.

\begin{tabular}{c|c|c|c|c}
\hline \hline Iteration & 0 & 5 & 10 & 20 \\
\hline$\zeta_{1}$ & 0.624 & 0.0852 & 0.1140 & 0.1000 \\
\hline$\zeta_{2}$ & 0.954 & 0.1952 & 0.2268 & 0.2000 \\
\hline$\tau$ & 16.473 & 18.5591 & 17.4625 & 17.0000 \\
\hline EFV & 8.6 & 1.6 & $2.4 \times 10^{-2}$ & $2.6 \times 10^{-15}$ \\
\hline EFC & 1 & 25 & 38 & 58 \\
\hline \hline
\end{tabular}

We see from Table 2 that, although the initial guess obtained from the randomization procedure is far from the optimum, good convergence is still achieved. The accuracy of our method is better than that obtained by the particle swarm optimization algorithm [9]. Moreover, the particle swarm algorithm in [9] needs to solve at least 900 dynamic systems. However, using our method, only $58 \times 4=232$ dynamic systems need to be solved. 


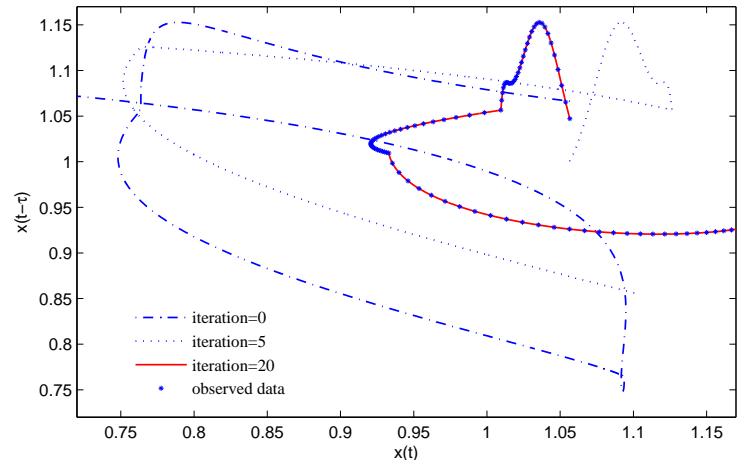

Fig. 4: Convergence of the output trajectory in Example 2.

\section{Conclusions}

This paper presents a gradient-based computational algorithm for solving the parameter identification problem for time-delay chaotic systems. In this algorithm, initial guesses for the unknown quantities (the magnitude of the time-delay and the values of unknown model parameters) are first chosen from a set of randomly-generated candidate solutions. Then, the gradients of the error function are computed by solving a set of auxiliary time-delay systems. On this basis, gradient-based optimization methods can be applied to solve the parameter identification problem. Simulation results on two classic time-delay chaotic systems show that, in comparison with existing methods, the proposed algorithm exhibits faster computation speed and higher accuracy.

\section{References}

[1] M. Yoshimoto, N. Morita, H. Shirahama, Chaotic oscillation of two-coupled chemical oscillators under time delay with an asymmetric condition, Chinese Journal of Physics, 51(2): 296-304, 2013.

[2] L. Junges, J. A. C. Gallas, Intricate routes to chaos in the Mackey-Glass delayed feedback system, Physics Letters A, 376(30-31): 2109-2116, 2012.

[3] J. A. Holyst, M. Zebrowska, K. Urbanowicz, Observations of deterministic chaos in financial time series by recurrence plots, can one control chaotic economy?, The European Physical Journal B - Condensed Matter and Complex Systems, 20(4): 531-535, 2001.

[4] C. M. Kim, W. H. Kye, S. Rim, S. Y. Lee, Communication key using delay times in time-delayed chaos synchronization, Physics Letters A, 333(3): 235-240, 2004.

[5] J. Sun, Delay-dependent stability criteria for time-delay chaotic systems via time-delay feedback control, Chaos, Solitons and Fractals, 21(1): 143-150, 2004.

[6] W. M. Bessa, A. S. de Paula, M. A. Savi, Adaptive fuzzy sliding mode control of a chaotic pendulum with noisy signal- s, ZAMM - Journal of Applied Mathematics and Mechanics, 94(3): 256-263, 2014.

[7] N. Li, J. F. Li, Y. P. Liu, Tracking control and parameter$\mathrm{s}$ identification of a class of chaotic systems with unknown parameters, Chinese Journal of Acta Physics Sinica, 60(5): 0050507-050507, 2011.

[8] S. Das, M. Srivastava, A. Y. T. Leung, Hybrid phase synchronization between identical and nonidentical threedimensional chaotic systems using the active control method, Nonlinear Dynamics, 73(4): 2261-2272, 2013.

[9] C. Dai, W. Chen, L. Li, Y. Zhu, Y. Yang, Seeker optimization algorithm for parameter estimation of time-delay chaotic systems, Physical Review E, 83(3): 036203, 2011.

[10] A. Alfi, Particle swarm optimization algorithm with dynamic inertia weight for online parameter identification applied to Lorenz chaotic system, International Journal of Innovative Computing, Information and Control, 8(2): 1191-1204, 2012.

[11] Y. Tang, X. Guan, Parameter estimation of chaotic system with time-delay: A differential evolution approach, Chaos, Solitons and Fractals, 42(5): 3132-3139, 2009.

[12] L. Belkoura, J. P. Richard, M. Fliess, Parameters estimation of systems with delayed and structured entries, Automatica, 45(5): 1117-1125, 2009.

[13] S. Diop, I. Kolmanovsky, P. E. Moraal, M. V. Nieuwstadt, Preserving stability/performance when facing an unknown time-delay, Control Engineering Practice, 9(12): 1319-1325, 2001.

[14] S. V. Drakunov, W. Perruquetti, J. P. Richard, L. Belkoura, Delay identification in time-delay systems using variable structure observers, Annual Reviews in Control, 30(2): 143-158, 2006.

[15] P. J. Gawthrop, M. T. Nihtila, Identification of time delays using a polynomial identification method, Systems and Control Letters, 5(4): 267-271, 1985.

[16] R. Loxton, K. L. Teo, V. Rehbock, An optimization approach to state-delay identification, IEEE Transactions on Automatic Control, 55(9): 2113-2119, 2010.

[17] L. Zunino, M. C. Soriano, I. Fischer, O. A. Rosso, C. R. Mirasso, Permutation-information-theory approach to unveil delay dynamics from time-series analysis, Physical Review E, 82(4), ID: 046212, 2010.

[18] N. U. Ahmed, Dynamic Systems and Control with Applications, Singapore: World Scientific, 2006.

[19] Q. Lin, R. Loxton, K. L. Teo, The control parameterization method for nonlinear optimal control: A survey, Journal of Industrial and Management Optimization, 10(1): 275-309, 2014.

[20] P. E. Gill, W. Murray, M. A. Saunders, SNOPT: An SQP algorithm for large-scale constrained optimization, SIAM Review, 47(1): 99-131, 2005.

[21] Q. Chai, R. Loxton, K. L. Teo, C. Yang, A unified parameter identification method for nonlinear time-delay systems, Journal of Industrial and Management Optimization, 9(2): 471486, 2013. 